\title{
Estudio mediante EIS de la respuesta electroquímica de la aleación AA5083 frente a señales DC anódicas
}

\author{
A. Aballe $\left(^{(*)}\right.$, M. Bethencourt ${ }^{(*)}$, F.J. Botana ${ }^{(*)}$, M. Marcos ${ }^{(* *)}$, J. Pérez-Mariscal ${ }^{(*)}$ y \\ M.A. Rodríguez-Chacón $\left.{ }^{*}\right)$
}

\begin{abstract}
Resumen Se ha llevado a cabo un estudio, mediante Espectroscopía de Impedancia Electroquímica (EIS), del comportamiento de la aleación AA5083 polarizada anódicamente en disolución de $\mathrm{NaCl}$ al 3,5\%. Los espectros de impedancia se registraron a distintos potenciales DC, seleccionados de acuerdo con el intervalo de pasividad que aparece en su curva de polarización anódica. En el rango de potenciales estudiado, se han obtenido tres tipos de respuesta electroquímica, cada una con un diagrama de $\mathrm{Ny}$ quist característico. Los diagramas obtenidos se han simulado construyendo los correspondientes circuitos equivalentes. La evolución, con la polarización aplicada, de los valores de los elementos que componen cada circuito ha permitido definir el intervalo de potencial en el que la capa pasiva es estable, se forman picaduras metaestables, o se nuclean y crecen picaduras. Por último, se propone utilizar "superficies de Bode" para visualizar globalmente la evolución del sistema con la polarización aplicada.
\end{abstract}

Palabras Clave: EIS. AA5083. Rama anódica. Pasividad. Corrosión por picaduras.

\section{EIS study of the electrochemical response vs anodic DC signals of AA5083 alloy}

\begin{abstract}
Electrochemical Impedance Spectroscopy (EIS) has been applied to characterise the behaviour of AA5083 alloy samples under anodic polarisation in a $3.5 \% \mathrm{NaCl}$ solution. EIS spectra were obtained at different DC potentials falling within the passivity range characteristic of the anodic polarisation branch of this alloy in the cited corrosive environment. Three different types of electrochemical response, with specific features in the corresponding Nyquist diagrams, were observed within the potential range covered in this study. The different type of spectra have been fitted with equivalent circuits. The evolution of the values of the different elements in the circuits as a function of the applied DC polarisation potential has been established. The analysis of these data has allowed us to determine the ranges of polarisation potentials where the passivation layer remains stable, metastable pits are nucleated or where stable pits grow. Finally, the use of a "Bode Surface Plot" has been proposed to get a better visualization of the evolution of the system with the applied DC potential.
\end{abstract}

Keywords: EIS. AA5083. Anodic branch. Passivity. Pitting corrosion.

\section{INTRODUCCIÓN}

En medios naturales, el aluminio presenta alta resistencia a la corrosión debido a la formación sobre su superficie de una película de óxido, de alto

(*) Dpto. de Ciencia de los Materiales e Ingeniería Metalúrgica y Química Inorgánica. Univ. de Cádiz. Fac. de Ciencias del Mar. Aptdo. 40. 11510-Puerto Real (Cádiz, España).e-mail: javier.botana@uca.es

(**) Dpto. de Ingeniería Mecánica y Diseño Industrial. Univ. de Cádiz. Esc. Sup. de Ingeniería. c/ Chile s/n. 11003-Cádiz (España). poder protector. Dicha película se desarrolla de forma casi inmediata al exponer el aluminio en atmósferas húmedas o en electrólitos acuosos neutros. En general, el comportamiento pasivo del aluminio está directamente relacionado con la naturaleza del electrólito donde se encuentra, dado que, tanto el espesor y la estructura de la capa pasiva como su estabilidad dependen de la composición de dicho electrólito y del potencial del sistema (1). Así, en medios que contienen iones agresivos, como, por ejemplo, iones cloruro, puede producirse la 
disolución del metal en zonas activas del mismo por la rotura de la película protectora (2). La estabilidad de dicha capa puede evaluarse desde un punto de vista electroquímico en función de los valores del potencial de nucleación de picaduras, $E_{\mathrm{np}}$, y del potencial de corrosión, $E_{\text {corr }}$ La diferencia entre ambos potenciales define el rango de pasividad del metal en el medio considerado (3). En disoluciones de $\mathrm{NaCl}$ al 3,5\%, para aluminio de alta pureza, este rango puede alcanzar unos $700 \mathrm{mV}$ (4).

Sin embargo, cuando se trata de aleaciones de aluminio, los precipitados asociados a la presencia de los elementos aleantes localiza las zonas activas sobre la superficie del material. Así, los compuestos intermetálicos que presentan un carácter activo con respecto a la matriz se comportan como zonas anódicas localizadas, sirviendo de núcleos para la formación de picaduras. Por el contrario, cuando los intermetálicos tienen carácter catódico, las picaduras se nuclean en las zonas de la matriz metálica que rodean a los mismos (5 y 6). En este último caso, con respecto al diagrama de polarización del aluminio puro se produce un aumento del potencial de corrosión permaneciendo, en muchos casos, prácticamente constante el potencial de nucleación de picaduras (7). De esa forma, se estrecha el rango de pasividad del material, llegándose en algunos casos a hacerse prácticamente nulo (5 y 8).

La espectroscopía de impedancia electroquímica (EIS) ha sido utilizada para estudiar el comportamiento de películas pasivas formadas sobre aluminio puro en medios de distinta agresividad (9-12). A partir de la respuesta electroquímica obtenida, en estos trabajos se establece el intervalo de potenciales en el que dichas películas son estables, así como los valores del potencial a partir de los cuales empieza a predominar el proceso de formación de picaduras. Sin embargo, en la bibliografía son escasos los trabajos en los que se aborden estudios similares sobre aleaciones de aluminio, debido a que, como se ha comentado anteriormente, la mayor parte de ellas presenta un rango de pasividad muy estrecho.

En este trabajo se ha realizado un estudio, mediante EIS, del comportamiento de la aleación de Al-Mg AA5083 polarizada anódicamente en el intervalo de potenciales limitado por los valores de $E_{\text {corr }}$ y $E_{\text {np }}$ característicos de este material en disolución acuosa de $\mathrm{NaCl}$ al 3,5\%. Aunque este intervalo puede considerarse estrecho, $50 \mathrm{mV}$, en relación con el del aluminio puro, resulta lo suficientemente amplio como para poder identificar mediante EIS las distintas etapas por las que transcurren los procesos de degradación de la película pasiva y de formación de picaduras.
TABLA. 1.- Composición nominal (\% en masa) de la aleación AA5083

TABLE. 1.- Composition of the AA5083 alloy (\% mass)

\begin{tabular}{|c|c|c|c|c|c|c|c|}
\hline $\mathrm{Mg}$ & $\mathrm{Mn}$ & $\mathrm{Si}$ & $\mathrm{Fe}$ & $\mathrm{Ti}$ & $\mathrm{Cu}$ & $\mathrm{Cr}$ & $\mathrm{Al}$ \\
\hline $4,0-4,0$ & $0,4-1,0$ & $0,4-0,7$ & 0,4 & 0,15 & 0,1 & $<0,25$ & Resto \\
\hline
\end{tabular}

\section{PROCEDIMIENTO EXPERIMENTAL}

Se han estudiado probetas de la aleación de Al$\mathrm{Mg}$ AA5083, de $30 \times 25 \times 4 \mathrm{~mm}$, cuya composición nominal se incluye en la tabla I. Antes de los ensayos, las muestras fueron sometidas a pulido húmedo en papel de $\mathrm{SiC}$ hasta 500 grits, desengrasadas con hexano y lavadas con agua destilada.

El rango de pasividad de la aleación en disolución aireada de $\mathrm{NaCl}$ al 3,5\% se determinó a partir de su curva de polarización cíclica (Fig. 1), con un potenciostato VersaStat de Parc; la velocidad de barrido empleada fue de $10 \mathrm{mV} / \mathrm{min}$, y como electrodo de referencia se empleó uno de $\mathrm{Ag} / \mathrm{AgCl}$. En estas condiciones, el valor del potencial de corrosión, $E_{\text {corr }}$, resultó ser $-0,755 \mathrm{~V}$ y el potencial de nucleación de picaduras, $E_{\mathrm{np}},-0,705 \mathrm{~V}$. Los resultados obtenidos indican, por una parte, que el potencial de nucleación de picaduras es aproximadamente el mismo que el del aluminio puro, mientras que la

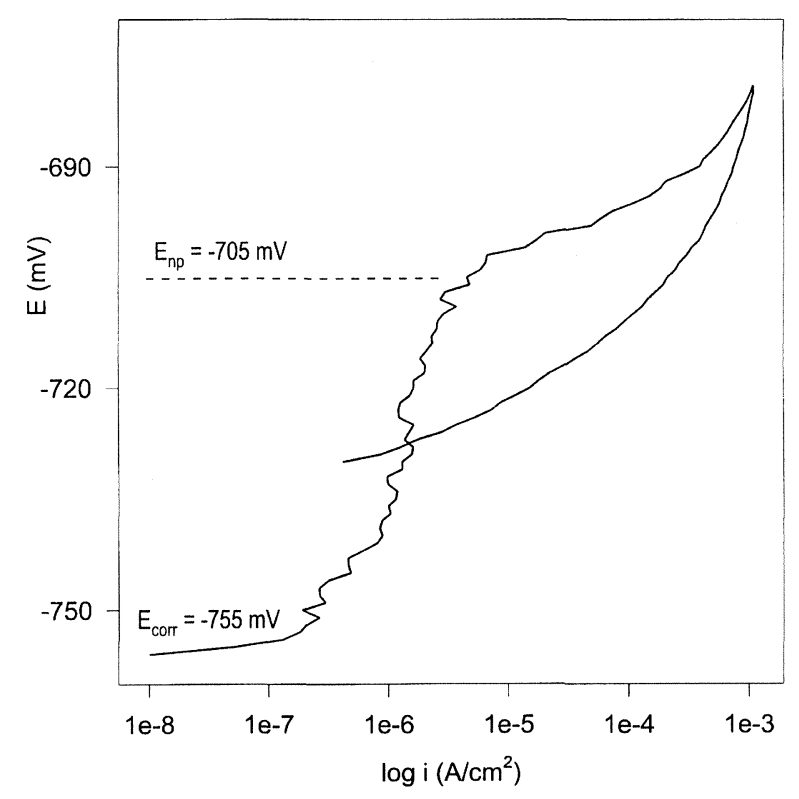

Fig. 1.- Curva de polarización cíclica de la aleación AA5083 en disolución acuosa aireada de $\mathrm{NaCl}$ al 3,5\%.

FIG. 1.- Cyclic polarisation curve corresponding to a AA5083 sample in $3.5 \% \mathrm{NaCl}$ aerated aqueous solution. 
presencia de los intermetálicos provoca una subida del potencial de corrosión. Todo ello trae consigo que el rango de pasividad de la aleación se reduzca a $50 \mathrm{mV}$. Estos resultados están en línea con los obtenidos por Galvele para otras aleaciones de aluminio (7).

De acuerdo con estos resultados, las medidas de impedancia se realizaron polarizando potenciostáticamente las muestras, entre 0 y $50 \mathrm{mV}$ por encima del potencial de corrosión. Antes de realizar el ensayo de impedancia, las muestras se polarizaron anódicamente, a cada uno de los potenciales seleccionados, hasta alcanzar valores estacionarios en la intensidad de corriente. Los espectros de impedancia se adquirieron utilizando un potenciostato SI 1287 acoplado a un analizador de frecuencia 1255 de Solartron, controlados ambos por ordenador haciendo uso de los programas CorrWare y Zplot, de Scribner. En general, la amplitud de la señal AC aplicada fue de $5 \mathrm{mV}$, excepto en aquellos experimentos en los que se polarizó el sistema a potenciales próximos al potencial de nucleación de picaduras. En estos casos, la amplitud se redujo a $2 \mathrm{mV}$ con objeto de garantizar la estabilidad del sistema. Los circuitos equivalentes que simulan la respuesta electroquímica del sistema se construyeron utilizando la rutina Equivalent Circuit del programa Zview, de Scribner.

\section{RESULTADOS Y DISCUSIÓN}

Dentro del intervalo de potenciales estudiado, se han obtenido tres tipos de respuesta electroquímica, caracterizadas cada una de ellas por presentar diagramas de Nyquist diferentes. Así, en la figura $2 a$ se muestran los diagramas de Nyquist obtenidos para polarizaciones de 10,20 y $30 \mathrm{mV}$, incluyéndose como referencia el diagrama registrado a circuito abierto. Como puede apreciarse, este último diagrama presenta dos arcos capacitivos, asociables a las interfases metal-óxido $(\mathrm{M} / \mathrm{O})$ y óxido-electrólito (O/E). De acuerdo con Brett (13), para sistemas en estado pasivo, el control del proceso se produce en la interfase $\mathrm{O} / \mathrm{E}$, por lo que su respuesta es la que predomina a bajas frecuencias. De este modo, el arco que aparece a altas frecuencias debe asociarse a los procesos de transferencia de cargas en la interfase $\mathrm{M} / \mathrm{O}$. En estas condiciones, la respuesta eléctrica del sistema puede simularse mediante un circuito como el de la figura $2 b$ (13 y 14). Los elementos de fase constante (CPE), incluidos en este circuito, permiten simular la depresión de los semicírculos capacitivos de la figura $2 a$. De acuerdo con (15), estas depresiones estarían asociadas a la existencia de irregularides en ambas interfases.
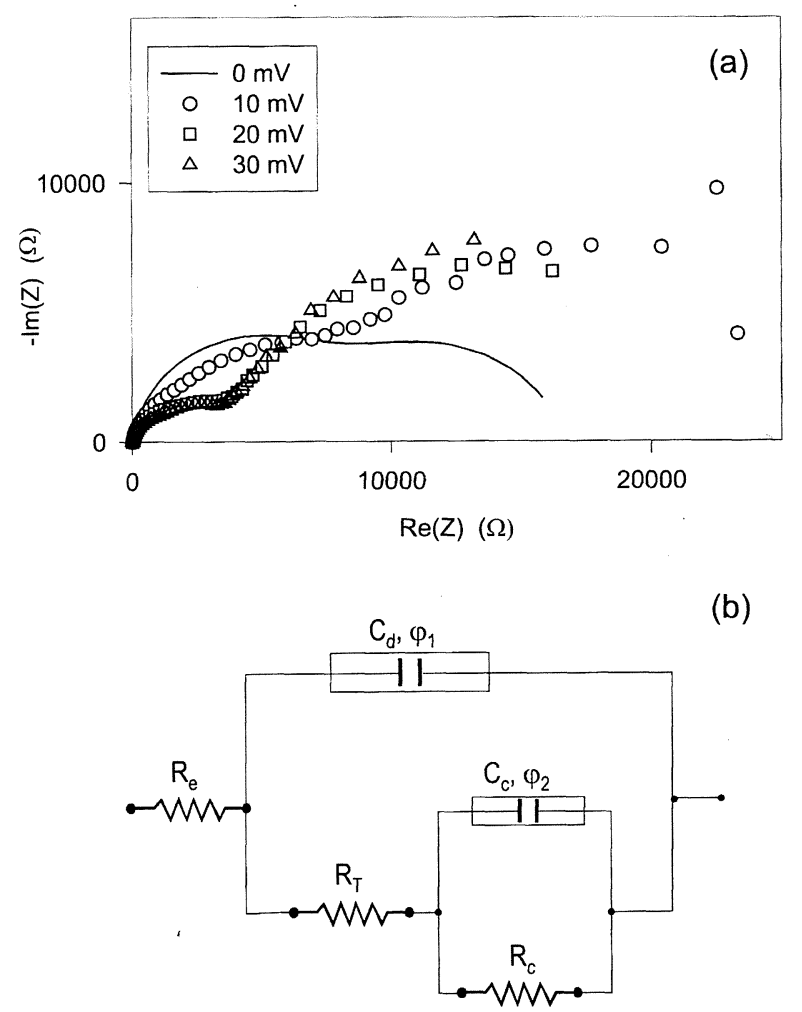

FIG. 2.- $-a$ ) Diagramas de Nyquist de la aleación AA5083 polarizada anódicamente a los potenciales indicados. Medio agresivo: disolución acuosa aireada de $\mathrm{NaCl}$ al 3,5 \%.b) Circuito equivalente.

FIG. 2.- a) Nyquist diagrams corresponding to AA5083 samples under anodic control at the referred potentials. Corrosive medium: $3.5 \% \mathrm{NaCl}$ aerated aqueous solution. b) Equivalent circuit.

Al polarizar anódicamente las muestras, se produce una deformación de los dos arcos capacitivos, dependiendo el grado de deformación del potencial aplicado. Así, para muestras polarizadas $10 \mathrm{mV}$, se produce el solapamiento de los dos arcos y un aumento de sus radios. Este último hecho indica que la polarización aplicada provoca un incremento en la resistencia de las dos interfases. Por otra parte, al analizar la evolución de los distintos elementos del circuito equivalente, se aprecia una disminución del valor de la capacidad asociada a la capa pasiva, $C_{\mathrm{C}}$, debido, posiblemente, a un aumento en su espesor. Por tanto, estos datos sugieren que al aplicar polarizaciones comprendidas entre 0 y $10 \mathrm{mV}$ se produce un aumento del efecto protector de la capa pasiva.

$\mathrm{Al}$ aumentar el nivel de polarización entre 20 y $30 \mathrm{mV}$, vuelven a definirse los arcos capacitivos y se reducen notablemente sus diámetros. En este intervalo, se produce el efecto contrario al comentado para $10 \mathrm{mV}$, es decir una disminución en la resistencia de ambas interfases y un aumento de $C_{\mathrm{C}}$. De acuerdo con (10 y 12), esta tendencia pone de 
manifiesto que, en tales condiciones, se inicia el proceso de degradación de la película pasiva.

En la figura $3 a$ se recogen los diagramas de $\mathrm{Ny}-$ quist obtenidos bajo polarizaciones de 40 y $50 \mathrm{mV}$. Como puede apreciarse, existen cambios notables con respecto a los que se representan en la figura $2 a$, siendo el aspecto más destacable la aparición de un lazo inductivo. De acuerdo con (10 y 16), la presencia de estos lazos puede deberse a la existencia de un proceso de corrosión por picaduras. Para incluir en el circuito equivalente el efecto de este tipo de procesos es necesario incorporar una malla $R L$.

En el diagrama obtenido a $40 \mathrm{mV}$, el lazo inductivo se inserta entre los dos arcos capacitivos (Fig.
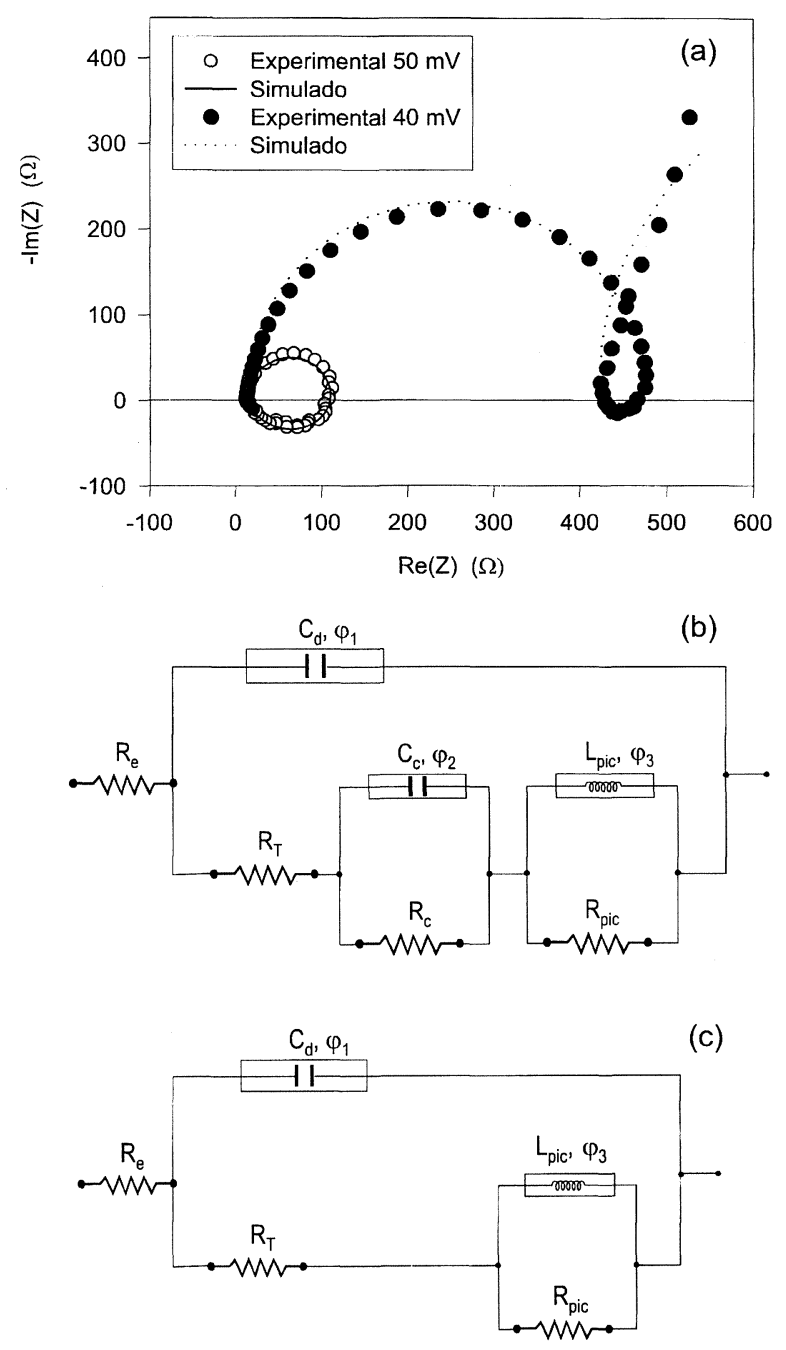

FIG. 3.- a) Diagramas de Nyquist de la aleación AA5083 polarizada anódicamente a los potenciales indicados. Medio agresivo: disolución acuosa aireada de $\mathrm{NaCl}$ al 3,5 \%.b) Circuito equivalente.

FIG. 3.- a) Nyquist diagrams corresponding to AA5083 samples under anodic control at the referred potentials. Corrosive medium: $3.5 \% \mathrm{NaCl}$ aerated aqueous solution. b) Equivalent circuit.
3 a). La permanencia del arco capacitivo de bajas frecuencias indica que, en estas condiciones de polarización, todavía existe la capa pasiva. Por esta razón, el circuito equivalente del sistema será el que se obtiene al incorporar, al de la figura $2 b$, una malla $R L$ (Fig. $3 b$ ). La coexistencia de la capa pasiva y de un proceso de corrosión localizada de baja intensidad es atribuida por algunos autores al carácter metaestable de las picaduras formadas $(9-12,16 \mathrm{y}$ 17).

En el diagrama registrado a $50 \mathrm{mV}$ (Fig. $3 a$ ), se observa, en primer lugar, la desaparición del arco capacitivo de bajas frecuencias. Esto indica que al aplicar este nivel de polarización se produce la disolución de la capa pasiva. En consecuencia, la malla $R C$ asociada a la misma debe ser eliminada del circuito equivalente (Fig. $3 c$ ). Por otra parte, el lazo inductivo aumenta de diámetro con respecto al que aparecía a $40 \mathrm{mV}$. De acuerdo con (10, 16 y 17), este tipo de diagramas es característico de un proceso catastrófico de corrosión por picaduras.

A partir de los diagramas de Nyquist resulta complicado obtener una visión de conjunto de la evolución de la respuesta eléctrica del sistema con el potencial DC aplicado. Esta dificultad surge de la diferencia existente entre los valores de los diámetros de cada uno de los arcos de los distintos diagramas. Para superar esta limitación, se propone construir una "superficie de Bode" a partir de la interpolación mediante algoritmos de Bézier (18) de los diagramas de Bode $|Z|-f$, tomando como dirección generatriz la correspondiente al eje en el que se ubica la polarización aplicada. De este modo, en la figura 4 , se ha representado la superficie de Bode correspondiente al comportamiento del sistema estudiado en condiciones de polarización anódica. El análisis del comportamiento del sistema debe efectuarse estudiando los cambios que se producen en

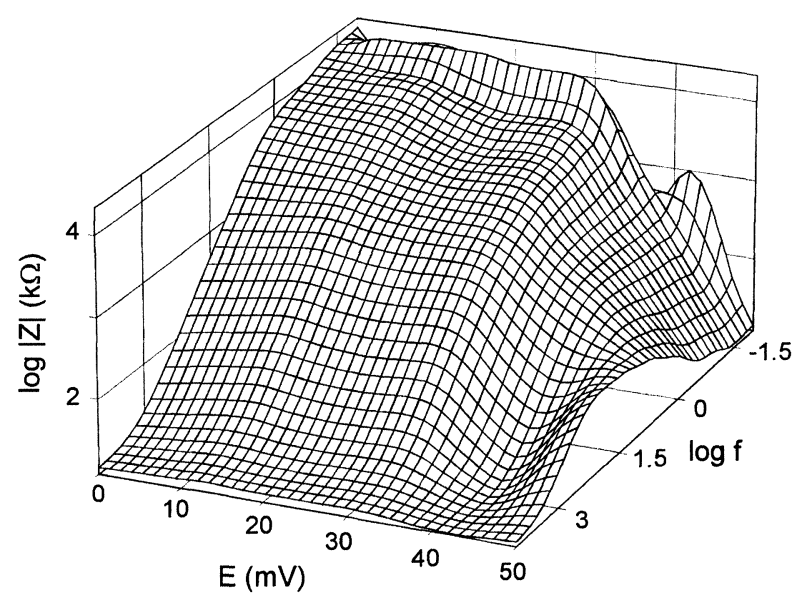

FIG. 4.- Superficie de Bode.

FIG. 4.-Bode surface plot. 
la forma de dicha superficie. Así, la geometría de la superficie de Bode en el intervalo de frecuencias más bajas es indicativa de la evolución de la velocidad de corrosión. Para el sistema estudiado puede observarse cómo, a partir de $10 \mathrm{mV}$, se produce un descenso del $|Z|$, lo cual implica un aumento de $v_{\text {corr }}$ para polarizaciones superiores a dicho valor. Por otra parte, los cambios de la forma con la polarización aplicada definen los valores del potencial para los que se verifican procesos de corrosión localizada. Así, en la figura 4, se observa cómo en torno al plano de $40 \mathrm{mV}$ se produce un cambio brusco en la forma de la superficie de Bode. Para polarizaciones de hasta $30 \mathrm{mV}$, se observan tramos ascendentes en la superficie de Bode, lo cual es indicativo de la coexistencia de dos interfases en el sistema. El inicio de la degradación de la capa pasiva a partir de 10 $\mathrm{mV}$ se detecta a través del descenso en el valor de $|Z|$ a las frecuencias más bajas. El proceso de corrosión por picaduras se manifiesta porque a partir de $40 \mathrm{mV}$ aparece un tramo descente a baja frecuencia en la superficie de Bode. Por último, el aumento de la intensidad del proceso de corrosión por picaduras con la polarización aplicada se manifiesta por el desplazamiento hacia frecuencias más altas en el valor máximo del $|Z|$.

\section{Agradecimiento}

Este trabajo ha sido financiado por la Comisión Interministerial de Ciencia y Tecnología (CICYT), Proyectos MAT97-1075-C03-01 y MAR95-2011.

\section{REFERENCIAS}

(1) Epelboin, I. y Keddam, M., en Passivity of Metals. Corrosion Monographs Series. (R.P. Frankenthal y J. Kruger, Eds.). The Electrochemical Soc. Nueva Jersey (EE.UU.). 1978: 184.
(2) MacDougall, B. y Graham, M.J., en Corrosion Mechanisms in Theory and Practice. (P. Marcus y J. Oudar, Eds.). Marcel Dekker, Nueva York (EE.UU.). 1995: 143.

(3) SzKLarsKa-SmialowsKa, Z. Pitting Corrosion of Metals. NACE, Houston, (TX., EE.UU.) 1986: 39.

(4) Venugopal, A. y Raja, V.S. Corros. Sci., 39 (12), 1997: 2.053 .

(5) Bethencourt, M., Botana, F.J., Calvino, J.J., Marcos, M., Pérez, J. y Rodríguez-Chacón, M.A. Mater. Sci. (En prensa).

(6) Aldykewicz, A.J., IsaAcs, H.S. y Davenport, A.J. Electrochem. Soc. 143 (1), 1996: 147.

(7) Galvele, J.R., De Micheli, S.M., Miller, I.L., Wexler, S.B. y Alanis, I.L. Critical Potentials for Localized Corrosion of Aluminium Alloys. (R. Staehle, B. Brown, J. Kruger y A. Agrawal, Eds.). NACE, Houston (TX, EE.UU.) 1947: 580.

(8) Hinton, B.R.W., Arnott, D.R. y Ryan, N.E. Mater. Forum, 9 (3), 1986: 162.

(9) Bessone, J.B., MAyer, C.E., JÜTner, K. y LOREnZ, W.J. Electrochim. Acta 28 (2), 1983: 171.

(10) Bessone, J.B., Salinas, D.R., Mayer, C.E., Eber, M. y LORENZ, W.J. Electrochim. Acta 37 (12), 1992: 2.283.

(11) Silverman, D.C. Corrosion, 46 (7), 1990: 589.

(12) DEWITT, J.H.W. y LENDERINK, H.W. Electrochim Acta, 41 (7-8), 1996: 1.111.

(13) BRetT, C.M. J. App. Electrochem. 20, 1990: 1.001.

(14) BRetT, C.M. Corros. Sci. 32 (2), 1992: 203.

(15) JÜTtNer, K., LoREnZ, W.J., Kendig, M.W. y MANSFeld, F. J. Electrochem. Soc, 135, 1988: 332.

(16) Sнiн, H. Corrosion, 52 (1), 1996: 43.

(17) Саbot, P.L., Garrido, J.A., Pérez, E., Moreira, A.H., Sumodjo, P.T.A. y Proud, W. Electrochim. Acta, 40 (4), 1995: 447.

(18) Farin, G. curves and Surfaces for CAGD. Academic Press. Londres (R.U.) 1995: 219. 